\title{
Plasmonic trapping with realistic dipole nanoantennas: Analysis of the detection limit
}

\author{
Andrea Lovera and Olivier J. F. Martin ${ }^{\mathrm{a})}$ \\ Nanophotonics and Metrology Laboratory, Ecole Polytechnique Fédérale de Lausanne, Lausanne 1015, \\ Switzerland
}

(Received 18 August 2011; accepted 19 September 2011; published online 11 October 2011)

\begin{abstract}
We use numerical simulations based on the surface integral technique to study the detection limit of plasmonic trapping with realistic dipole antennas. The induced plasmon resonance shift due to the coupling between an antenna and a nanoparticle is studied for different antennas geometries, different positions, sizes, and materials for the trapped nanoparticle. The shift of the antenna resonance is found to be linear with the near-field intensity enhancement caused by the antenna and further dependents on the volume and refractive index of the trapped nanoparticle. Detection limit of $5 \mathrm{~nm}$ for plasmonic particles and $6.5 \mathrm{~nm}$ for high index dielectrics is reported. (C) 2011 American Institute of Physics. [doi:10.1063/1.3650267]
\end{abstract}

After the demonstration of optical trapping, ${ }^{1}$ a lot of efforts have been invested to improve its performances. ${ }^{2,3}$ However, fundamental limitations of this technique make the far-field trapping of objects smaller than the diffraction limit difficult to achieve. The utilization of near-field optics can, however, overcome this limitation; ${ }^{4}$ in particular, the extremely confined electromagnetic field produced by plasmonic resonant nanostructures seems very well suited to trap nanoparticles (NPs) and biological entities. ${ }^{5-7}$ Furthermore, this technique offers other advantages like reduced laser power, ${ }^{8}$ simpler optics, ${ }^{9}$ and possible integration with analytical techniques like surface-enhanced Raman spectroscopy (SERS). ${ }^{10}$

For NPs much smaller than the diffraction limit, trapping events cannot be tracked by direct observation and a different approach based on the detection of the interaction between the trap and the object must be used. ${ }^{8,9} \mathrm{~A}$ useful property of plasmonic nanostructures is indeed their high sensitivity with respect to variations of the surrounding refractive index. ${ }^{11}$ In this letter, we will focus on trapping and detection performed with dipole antennas since they show good performances in terms of field enhancement ${ }^{12}$ and the properties of their localized surface plasmon resonances (LSPR) can be easily engineered. ${ }^{13}$

Even if the detection is based on far-field measurements, a deep knowledge of the near-field is required to understand the properties of the system. In fact, the trapping potential as well as the shape of the field enhancement, strongly depend on the actual geometry of the nanoantennas. It has been shown that despite the scattering cross-section of an ideal antenna being similar to that of a realistic one, the near-field intensity distribution can be quite different and must be taken into account for a more accurate analysis. ${ }^{14}$ For this reason, we have performed numerical simulations based on the surface integral equation approach $(\mathrm{SIE})^{15}$ using realistic geometries for modeling the nanoantennas, Fig. 1(a). Scanning electron microscope pictures of fabricated antennas were

\footnotetext{
a) Author to whom correspondence should be addressed. Electronic mail: olivier.martin@epfl.ch.
}

collected and treated in order to obtain a 3D model of the antennas. The proposed method is very powerful since it allows to discretize the surface of an arbitrary shape object following the real geometry without introducing unrealistic sharp corners. A detailed analysis of the optical forces involved in this study is beyond the scope of this letter and we refer the reader to references. ${ }^{9,16-18}$

The detection of trapping events is done by monitoring in time the scattering cross-section of a single antenna. ${ }^{9}$ If the antenna is not perturbed, the LSPR remains constant in time. However, if an object is trapped by the antenna, the local refractive index changes and, as a consequence, the LSPR peak shifts. Figure 1(b) shows the scattering crosssections simulated with the SIE method for the antenna in Fig. 1(a) with a $15 \mathrm{~nm}$ gold NP placed at different positions around the antenna. All simulations were performed in water with a constant antenna arm length of $100 \mathrm{~nm}$. The result of the coupling between the NP and the antenna is a red-shift of the antenna LSPR. The shift magnitude is not constant with the NP position. It vanishes for particles placed laterally, position $\mathrm{D}$ in Fig. 1(b), while it is maximum for a particle in the center of the nanogap (A) and relatively small for particles at the end (B) or on the side of the gap (C). It is evident that the shift, as also highlight by Fig. 1(c), strongly depends on the position of the NP with respect to the near-field distribution generated by the antenna. In general, higher is the field, greater is the shift. This result is in agreement with previous studies on LSPR sensors where the figure of merit for the sensitivity is proportional to the field confinement in the plasmonic structure. ${ }^{11,19,20}$ Figure 1 (c) shows a $15 \mathrm{~nm}$ gold NP crossing a realistic antenna along the gap, with position zero at the center of the structure. When the NP is far away, there is no interaction with the antenna and so the scattering cross-section is not perturbed. Moving closer, the two objects start to feel each other and couple. An analysis of these data shows that the shift profile is linear with the near-field intensity at the NP location in the absence of the NP. To prove this statement, the same simulations were performed with an ideal antenna composed by two cuboids facing each other. For this geometry, the field profile does not increase 

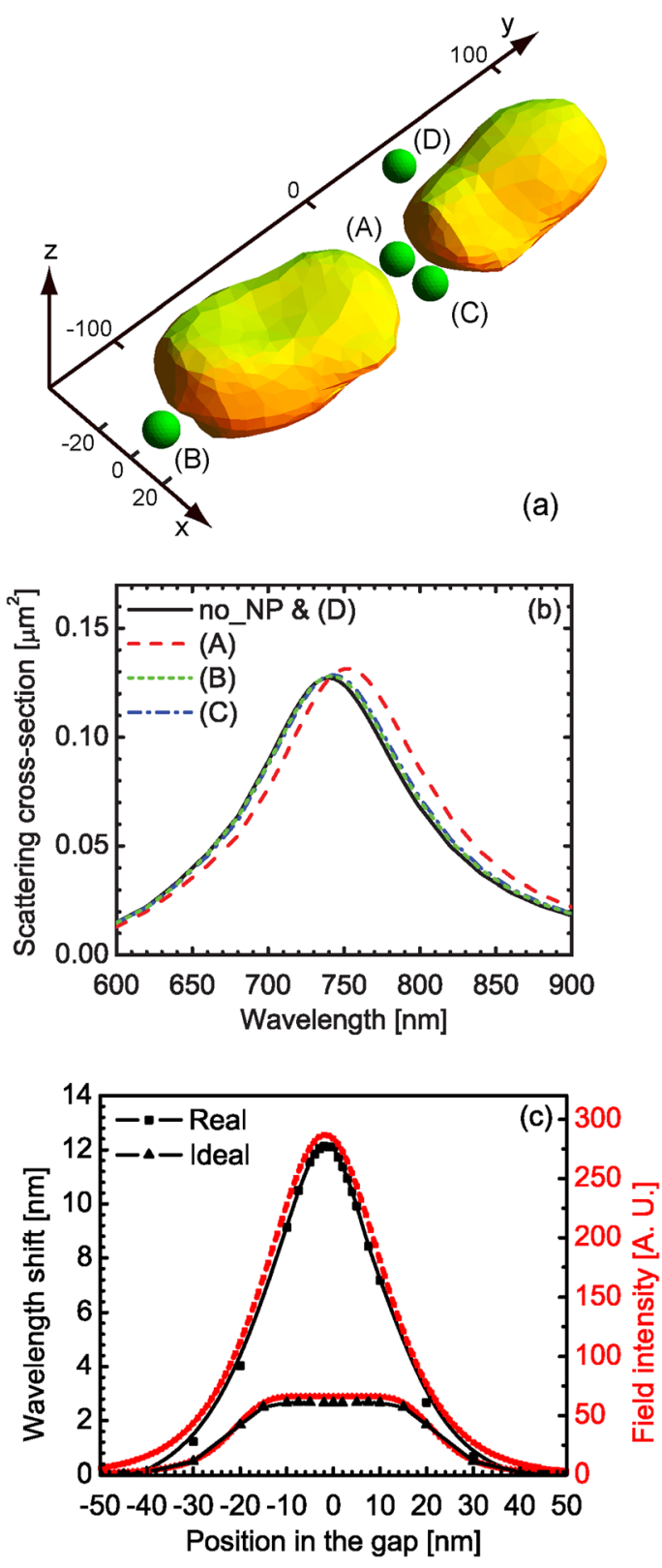

FIG. 1. (Color online) (a) 3D rendering of a realistic dipole antenna with gold NPs placed in different locations. (b) Scattering cross-section for the antenna in (a) with a $15 \mathrm{~nm}$ NP located (A) in the center of the gap, (B) at one end, (C) on one side, (D) laterally in the gap. An antenna without NP exhibits the same scattering cross section as that corresponding to (D). The coupling causes a red-shift of the LSPR which strongly depends on the position of the NP. (c) Peak wavelength shift and field intensity as a function of the position along the $\mathrm{x}$ axis $(\mathrm{y}=0)$ caused by a $15 \mathrm{~nm}$ gold NP for the antenna in (a) and for an ideal dipole antenna (shift scaled by a factor of $1 /$ 3). The shift is linear with the near-field intensity felt by the NP. continuously in the gap but saturates in the central region and so does the wavelength shift, Fig. 1(c). The same applies for the optical forces generated by the two geometries (realistic and ideal) where much better spatial confinement is obtained with the former, compared to the latter, although their trap stiffness is of the same order of magnitude. These results further emphasize the importance of using a realistic geometry when comparing simulations and experiments.

This linear dependence of the shift on the near-field intensity has a general validity and can be also applied to the case where a NP lies close to one end of the antenna. In these locations, the field is also enhanced sufficiently to generate a stable trapping potential, ${ }^{9}$ but the relative shift is much smaller than when the NP is trapped in the gap. This is a further indication that links the shift magnitude with the nearfield enhancement intensity.

Figures 2(a) and 2(b) present SIE simulations for realistic antennas with spherical NPs in the center of the gap. Two cases are considered, $15 \mathrm{~nm}$ and $25 \mathrm{~nm}$ gaps. The LSPR shift is shown as a function of the particle volume and refractive index. As expected, the shift increases with the NP diameter and follows a quadratic behavior for plasmonic NPs. It is, however, more linear for dielectrics and shows as well a dependence on the refractive index. The difference between metals and dielectrics arises from the fact that in the first case, the shift can be caused by hybridization between the modes of the trapped plasmonic NP and the trapping plasmonic structure; ${ }^{21}$ while in the second case, it originates from a local variation of the refractive index. Comparing the same simulations for different gap sizes, one can see that smaller is the gap, the larger is the shift. This is a consequence of the higher field enhancement in small gap antennas. When the NP diameter goes below $5 \mathrm{~nm}$, the shift decreases below $1 \mathrm{~nm}$ for antennas with a $25 \mathrm{~nm}$ gap and below $2 \mathrm{~nm}$ for a $15 \mathrm{~nm}$ gap; as a result, the sensing becomes more difficult especially for low refractive index dielectrics. The same conclusion can be applied to NPs trapped at one extremity, Fig. 2(c). However, in this case, the detection is more difficult since the shifts are in general much smaller but still possible for plasmonic nanoparticles larger than $15 \mathrm{~nm}$ in diameter and high index dielectrics $20 \mathrm{~nm}$ and larger.

These results are summarized in Fig. 3 which shows the shift as a function of the gap size. For increasing gap, the shift is reduced since the field enhancement is lower. In general, if one wishes to maximize the sensitivity, the gap size should be comparable to the size of the object to be analyzed. Anyway, for plasmonic particles larger than $20 \mathrm{~nm}$,
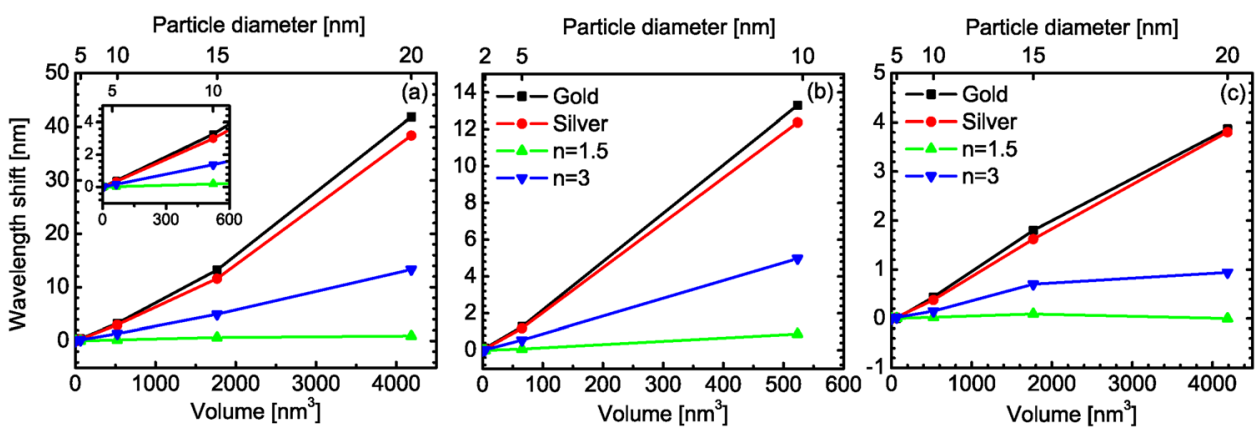

FIG. 2. (Color online) Shift of the LSPR as a function of the NP volume and material for (a) $25 \mathrm{~nm}$ gap and NPs in the center, position A in Fig. 1(a); (b) $15 \mathrm{~nm}$ gap and NPs in the center (position A); and (c) $25 \mathrm{~nm}$ gap and NPs at one extremity (position B). The highest sensitivity is obtained for the case (b), where the field intensity is greatest. 


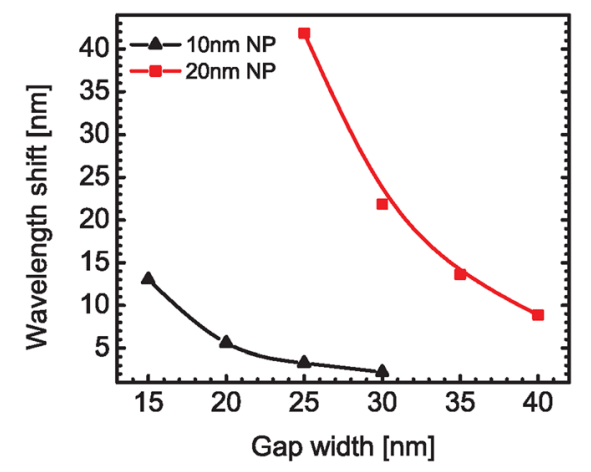

FIG. 3. (Color online) Shift of the LSPR as a function of the gap width for different gold NPs placed in the center, position A in Fig. 1(a). The sensitivity increases with decreasing gap size.

the shift remains so important that detection is easy, even with very large gaps. On the other hand, for detecting very small particles, the gap should be as small as possible, which can prove a nanofabrication challenge below $10 \mathrm{~nm}$.

In conclusion, we have numerically studied the detection limit of plasmonic trapping with dipole antennas. With this technique, plasmonic particles with diameter down to $5 \mathrm{~nm}$ and high index dielectric nanoparticles down to $6.5 \mathrm{~nm}$ can be detected. However, small and low index dielectric particles (including biological entities) remain difficult to detect directly.
This work was supported by the European Community's Seventh Framework Program (FP7-ICT-2009-4, Grant agreement 248835).

${ }^{1}$ A. Ashkin, Phys. Rev. Lett. 24, 156 (1970).

${ }^{2}$ D. G. Grier, Nature 424, 810 (2003).

${ }^{3}$ K. C. Neuman and S. M. Block, Rev. Sci. Instrum. 75, 2787 (2004).

${ }^{4}$ D. Erickson, X. Serey, Y.-F. Chen, and S. Mandal, Lab Chip 11, 995 (2011).

${ }^{5}$ M. Righini, A. S. Zelenina, C. Girard, and R. Quidant, Nat. Phys. 3, 477 (2007).

${ }^{6}$ L. Huang, S. Maerkl, and O. J. F. Martin, Opt. Express 17, 6018 (2009).

${ }^{7}$ M. L. Juan, R. M. Righini, and R. Quidant, Nat. Photon. 5, 349 (2011).

${ }^{8}$ M. L. Juan, R. Gordon, Y. J. Pang, F. Eftekhari, and R. Quidant, Nat. Phys. 5, 915 (2009).

${ }^{9}$ W. Zhang, L. Huang, C. Santschi, and O. J. F. Martin, Nano Lett. 10, 1006 (2010).

${ }^{10}$ A. Lovera and O. J. F. Martin, Proc. SPIE 8097, 80971Q (2011).

${ }^{11}$ J. N. Anker, W. P. Hall, O. Lyandres, N. C. Shah, J. Zhao, and R. P. Van Duyne, Nat. Mater. 7, 442 (2008).

${ }^{12}$ P. Muhlschlegel, H.-J. Eisler, O. J. F. Martin, B. Hecht, and D. W. Pohl, Science 308, 1607 (2005).

${ }^{13}$ H. Fischer and O. J. F. Martin, Opt. Express 16, 9144 (2008).

${ }^{14}$ A. M. Kern and O. J. F. Martin, Nano Lett. 11, $482(2011)$.

${ }^{15}$ A. M. Kern and O. J. F. Martin, J. Opt. Soc. Am. A 26, 732 (2009).

${ }^{16}$ M. Nieto-Vesperinas, P. C. Chaumet, and A. Rahmani, Phil. Trans. R. Soc. A 362, 719 (2004).

${ }^{17}$ L. Huang and O. J. F. Martin, Opt. Lett. 33, 3001 (2008).

${ }^{18}$ V. D. Miljkovic, T. Pakizeh, B. Sepulveda, P. Johansson, and M. Kall, J. Phys. Chem. C 114, 7472 (2010).

${ }^{19}$ L. J. Sherry, S.-H. Chang, G. C. Schatz, R. P. Van Duyne, B. J. Wiley, and Y. Xia, Nano Lett. 5, 2034 (2005).

${ }^{20}$ A. Unger and M. Kreiter, J. Phys. Chem. C 113, 12243 (2009).

${ }^{21}$ E. Prodan, C. Radloff, N. J. Halas, and P. Nordlander, Science 302, 419 (2003). 